\title{
ASOCIACIONES PALINOLÓGICAS CON ANGIOSPERMAS EN EL CRETÁCICO SUPERIOR DE LA CUENCA NEUQUINA, ARGENTINA
}

\author{
PATRICIA VALLATI \\ Laboratorio de Bioestratigrafía, UNPSJB, Ciudad Universitaria, 9000, Comodoro Rivadavia, Chubut, Argentina. \\ patricia.vallati@gmail.com
}

\begin{abstract}
PALYNOLOGICAL ASSEMBLAGES WITH ANGIOSPERMS IN THE UPPER CRETACEOUS OF THE NEUQUÉN BASIN, ARGENTINA. Two new palynological assemblages including angiosperm pollen grains from Upper Cretaceous outcrops in the Neuquén Basin (west-central Argentina) are characterized and their biogeographic relationships are interpreted. The first one of the microfloras, from the stratigraphic unit Bajo de la Carpa, in the Neuquén Group at El Zampal, south of Mendoza, is characterized by the Confossia vulgaris-Cretacaeiporites scabratus pair, and it is assigned to the Santonian-?lower Campanian chronostratigraphic interval. This assemblage includes some elements recorded in Brazil and Africa, and it seems to belong to a transitional belt between the Equatorial and Austral paleofloristic provinces. The second one, from the Allen Formation at Lomas Coloradas, northeast of Neuquén province, is characterized by the Tricesticillus tetris-Peninsulapollis gilli pair, and it is assigned to the Campanian-Maastrichtian interval. Several representative components of the latter assemblage are well represented in Australia and the Antarctic Peninsula. The new information provided by these microfloras extends the palynological record, within the framework of a developing project that involves Cretaceous assemblages with angiosperms in central and septentrional Patagonia.
\end{abstract}

Key words: angiosperm pollen, Upper Cretaceous, Neuquén Basin, Argentina, paleobiogeography.

\begin{abstract}
RESUMO - Este trabalho visa caracterizar duas novas associações palinológicas que incluem grãos de pólen de angiospermas, procedentes de afloramentos do Cretáceo Superior da bacia Neuquina (centro-oeste da Argentina). A primeira microflora provém da unidade estratigráfica Bajo de la Carpa, pertencente ao Grupo Neuquén na localidade de El Zampal (sul de Mendoza), e é caracterizada pelo par Confossia vulgaris-Cretacaeiporites scabratus, sendo atribuída ao intervalo cronoestratigráfico Santoniano-Campaniano inferior?. Essa associação inclui elementos registrados no Brasil e na África e parece pertencer a uma faixa de transição entre as províncias paleoflorísticas Equatorial e Austral. A segunda microflora, procedente da Formação Allen em Lomas Coloradas (nordeste da Província de Neuquén), está caracterizada pelo par Tricesticillus tetris-Peninsulapollis gilli e é atribuída ao Campaniano-Maastrichtiano. Vários componentes representativos desta associação estão bem representados na Austrália e na Península Antártica. A nova ocorrência ora apresentada amplia o registro palinológico de associações paleoflorísticas do Cretáceo com angiospermas na Patagônia central e setentrional argentina.
\end{abstract}

Palavras-chave: grãos de pólen, angiospermas, Cretáceo Superior, bacia Neuquina, Argentina, paleobiogeografia.

\section{INTRODUCCIÓN}

Entre los trabajos pioneros relacionados con la palinología de las angiospermas en Patagonia se destaca el registro de elementos primitivos como Clavatipollenites y Asteropollis (Archangelsky \& Gamerro, 1967; Archangelsky et al., 1983, 1984) en el ámbito del Macizo del Deseado, Cuenca Austral, y la asociación que incluye elementos asignados a Huitrinipollenites y Stephanocolpites junto a granos tricolpados, reportada por Volkheimer \& Salas (1975) en la Cuenca Neuquina.

La presente contribución continúa estudios palinológicos previos de asociaciones cretácicas con angiospermas en Patagonia central y septentrional (Vallati, 1995, 2001a, 2002a,b, 2006). En dicho marco, la presencia de Tucanopollis crisopolensis es citada, por primera vez en Argentina, en la Formación D-129, Cuenca del Golfo San Jorge (Vallati, 2006).
Este marcador paleoecuatorial se incluye en una microflora con granos monosulcados de angiospermas basales (Zona de Clavatipollenites hughesii del Barremiano tardío?/Aptiano temprano) (Vallati, 2001b). Asimismo, en la Cuenca Neuquina, en la localidad Bajada del Agrio y en los niveles basales del Grupo Neuquén, en la localidad El Zampal, se describieron tres asociaciones palinológicas que incluyen el primer registro y posterior diversificación de las angiospermas en las localidades estudiadas. Las mismas corresponden a la Zona de Afropollis zonatus del Aptiano con granos relativamente primitivos y la Zona de Fraxinoipollenites fragilis y el Nivel con Cretacaeiporites polygonalis, asignados al Cenomaniano, con granos tricolpados y tricolporados de eudicotiledóneas (Vallati, 2001a,b, 2002a). Las microfloras ahora presentadas fueron recuperadas en depósitos predominantemente continentales del Cretácico Superior de la mencionada Cuenca Neuquina en las localidades de El Zampal y Lomas Coloradas (Figura 1). 


\section{ASPECTOS GEOLÓGICOS}

En esta contribución, la sucesión sedimentaria continental bien expuesta en la localidad El Zampal se incluye en el Grupo Neuquén emend. Uliana \& Dellapé (1981). Las referencias previas a los "Estratos con Dinosaurios" (Vallati, 2001a,b, 2002a,b, 2006; Musacchio \& Vallati, 2007) deberían ser consideradas en sinonimia con el Grupo Neuquén emend. Uliana \& Dellapé (1981). Para una discusión de la terminología adoptada para estas capas ver Musacchio (2010).

Las distintas unidades estratigráficas que integran el Grupo Neuquén han aportado numerosos restos fósiles de vertebrados. En particular, y dentro de las areniscas de la Formación Bajo de la Carpa, de interés en esta contribución, se han encontrado restos de reptiles (dinosaurios y crocodyliformes) y aves (ver Leanza et al., 2004). Estudios micropaleontológicos que incluyen asociaciones de microfósiles calcáreos (Musacchio, 1973, 1989, 2006, 2010; Musacchio \& Vallati, 2007) y palinológicas (Vallati, 2001a, 2002a,c) en distintos niveles del Grupo Neuquén, han aportado evidencias cronológicas y/o ambientales para las unidades portadoras.

En la denominada Cuenca de Añelo, la parte superior del Grupo Neuquén incluye las entidades litoestratigráficas Bajo de la Carpa y Anacleto (Cazau \& Uliana, 1973; Uliana \& Dellapé, 1981; Ramos, 1981; Leanza et al., 2004). La primera abunda en areniscas de origen fluvial y la segunda en fangolitas varicolores que representan un evento lacustre de gran extensión regional. En El Zampal (aproximadamente $69^{\circ} 33^{\prime} \mathrm{O}, 36^{\circ} 32^{\prime} \mathrm{S}$ ), y a diferencia de lo que ocurre en otras localidades de la cuenca mencionada, estas unidades litoestratigráficas de la parte superior carecen de identidad como formaciones y son tratadas como miembros por Uliana \& Musacchio (1978) (ver también Figuras 2-4, este trabajo).

Las areniscas que suprayacen a las fangolitas rojas de la Formación Plottier, fueron correlacionadas por Uliana (Uliana \& Musacchio, 1978) con la Formación Bajo de la Carpa Herrero Ducloux, que incluye areniscas varicolores dominantes con algunas intercalaciones de pelitas (Figura 2). Precisamente en uno de estos niveles pelíticos, en la
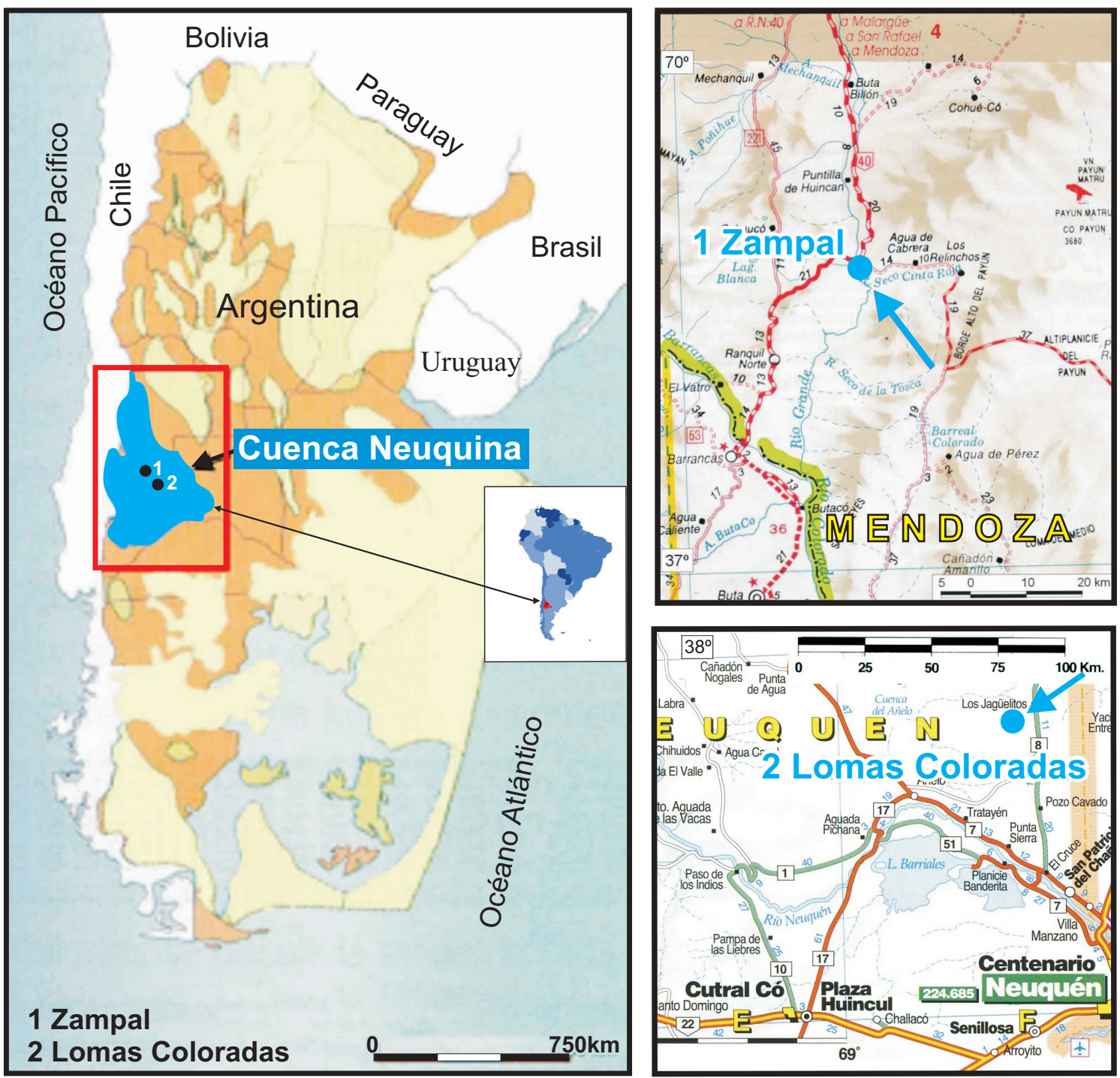

Figura 1. Mapa de ubicación de las localidades estudiadas (modificado de Veiga et al., 1999).

Figure 1. Location map of the studied sites (modified from Veiga et al., 1999). 
parte cuspidal de la entidad, se recuperó la microflora ahora presentada (Figura 4).

El Grupo Malargüe (Gerth, 1925), definido para los afloramientos próximos al Arroyo Loncoche en Mendoza, se corresponde con el conjunto de las formaciones Allen, Jagüel y Roca. Estas últimas tienen, en cambio, identidad en las provincias de Neuquén y Río Negro.

El mencionado grupo se dispone en disconformidad sobre las capas terminales del Grupo Neuquén. La microflora estudiada procede de la Formación Allen sensu Uliana \& Dellapé (1981), en la localidad Lomas Coloradas (aproximadamente $\left.68^{\circ} 28^{\prime} \mathrm{O}, 38^{\circ} 04^{\prime} \mathrm{S}\right)$, próximo al neo-estratotipo para esta unidad propuesto por Uliana \& Dellapé (1981) (Figura 5). La entidad litoestratigráfica incluye areniscas en la base, pelitas intermedias y yeso en la parte superior (Figura 6).

Con anterioridad, Palamarczuk y Gamerro (1988), reportaron una asociación palinológica con fitoplancton (Botryococcus, Pediastrum y escasos quistes de dinoflagelados) y esporas de helechos heterosporados proveniente del mencionado neoestratotipo. En distintos afloramientos de esta unidad, se han registrado restos fósiles que incluyen peces, plesiosaurios (Ellasmosauridae), dinosaurios y más recientemente mamíferos (Salgado et al., 2007; Rougier et al., 2009).

\section{MATERIAL Y MÉTODOS}

Se procesaron cuatro muestras (Z51 a Z54) provenientes de intercalaciones pelíticas en la parte superior de la entidad estratigráfica Bajo de la Carpa, en El Zampal (Figuras 1, 2,4).
Sólo una muestra (Z54) resultó fértil y su contenido polínico es presentado en esta contribución. A su vez en la localidad Lomas Coloradas (Figura 1) se recolectaron siete muestras (383 a 389) en los niveles pelíticos de la sección intermedia de la Formación Allen (Figura 6). En seis de estas muestras se recuperaron palinomorfos en buen estado de preservación que permitieron el reconocimiento de la asociación palinológica caracterizada por el par Tricesticillus tetrisPeninsulapollis gilli.

Las muestras fueron procesadas según el procedimiento palinológico estándar, que incluye la remoción de los carbonatos con ácido clorhídrico y de los silicatos con ácido fluorhídrico y el posterior lavado del residuo con ácido clorhídrico concentrado a $90^{\circ} \mathrm{C}$. En algunos casos fue necesaria una oxidación breve con ácido nítrico. El residuo orgánico fue finalmente montado en los portaobjetos con glicerina-gelatina. Las muestras fueron estudiadas con un microscopio Carl Zeiss KF 2. Las fotomicrografías fueron obtenidas con una cámara digital Nikon Coolpix P2.

\section{Repositorio del material}

El material estudiado pertenece a la colección de micropaleontología del Laboratorio de Bioestratigrafía de la Universidad Nacional de la Patagonia San Juan Bosco, donde se encuentran archivados con la sigla CR.P.CV. Los especímenes se identifican con el número de slide correspondiente, junto con las coordenadas que corresponden a la Escala Vernier del Microscopio Carl Zeiss. En el Apéndice se incluye la lista de especies ilustradas y/o mencionadas en el texto.

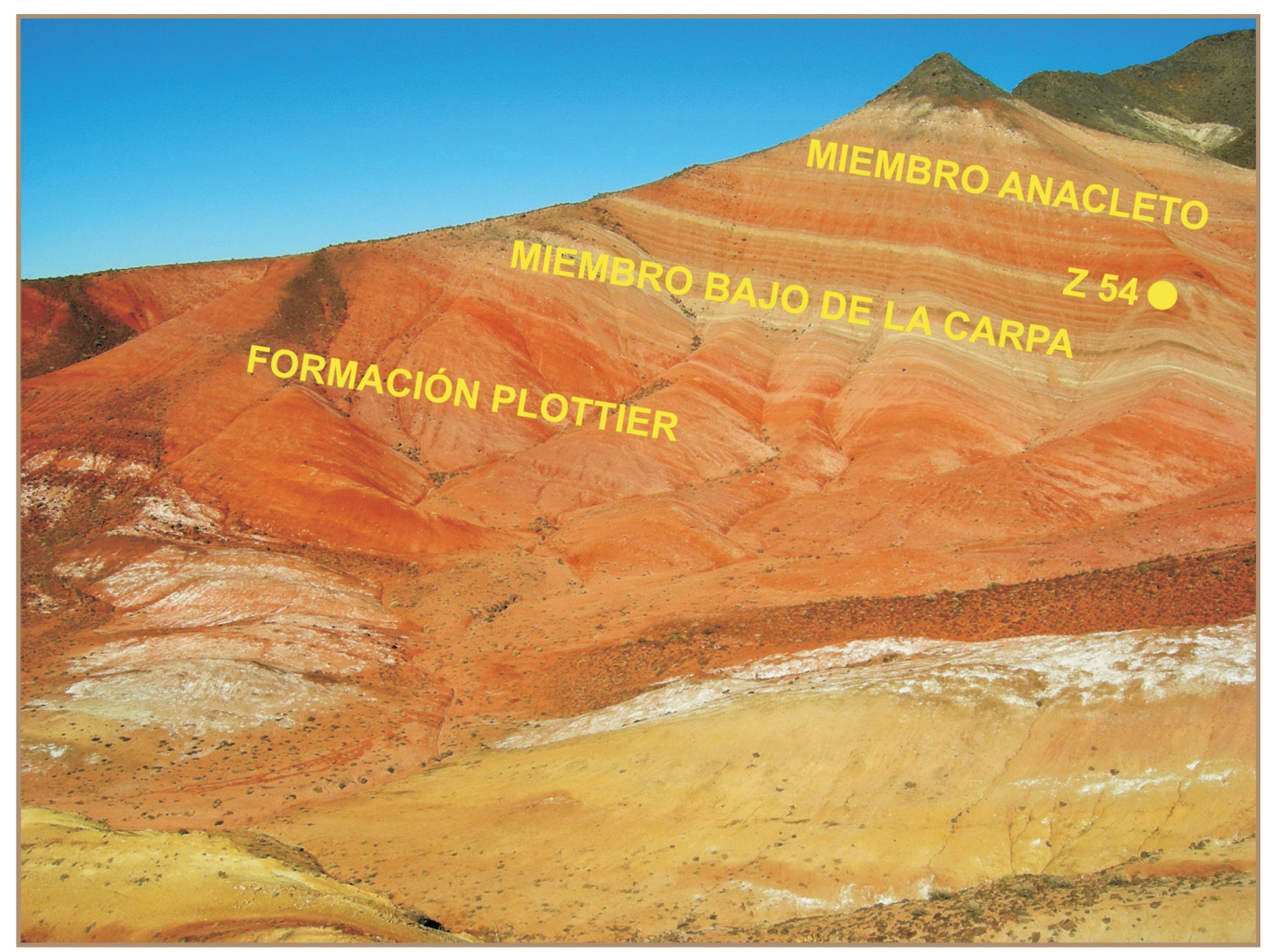

Figura 2. Vista panorámica de la parte superior del Grupo Neuquén, con indicación del nivel fértil (Z54). Sin escala.

Figure 2. Panoramic view of the upper part of the Neuquén Group indicating the fertile level (Z54). Not on scale. 


\section{CARACTERIZACIÓN DE LAS ASOCIACIONES PALINOLÓGICAS}

\section{Microflora de Bajo de la Carpa}

La asociación de Bajo de la Carpa representa una comunidad vegetal dominada por gimnospermas y en particular por coníferas. Los granos porados de Classopollis representan cerca del $80 \%$ de los componentes terrestres de la asociación. Otros granos de gimnospermas bien representados en la microflora son los bisacados. Por su parte, el polen poliplicado, si bien no es abundante al considerar la participación a nivel supragenérico $(2 \%)$, se presenta diversificado, con cuatro géneros (Gnetaceaepollenites,
Steevesipollenites, Equisetosporites, Singhia) y varias especies. El predominio de granos de Classopollis (Cheirolepidaceae) y la diversificación de los poliplicados (Ephedraceae) sugieren condiciones de aridez para la flora.

La asociación de angiospermas incluye granos monosulcados, tricolpados, tricolporoidados, tricolporados, triporados y periporados. Algunos taxa están escasamente representados, en algunos casos por un solo ejemplar, dificultando las determinaciones a nivel específico.

Esta microflora está caracterizada por la presencia de Confossia vulgaris y Cretacaeiporites scabratus, dos taxa de angiospermas característicos del Cretácico Superior de la Región Ecuatorial (Herngreen et al., 1996). Confossia vulgaris

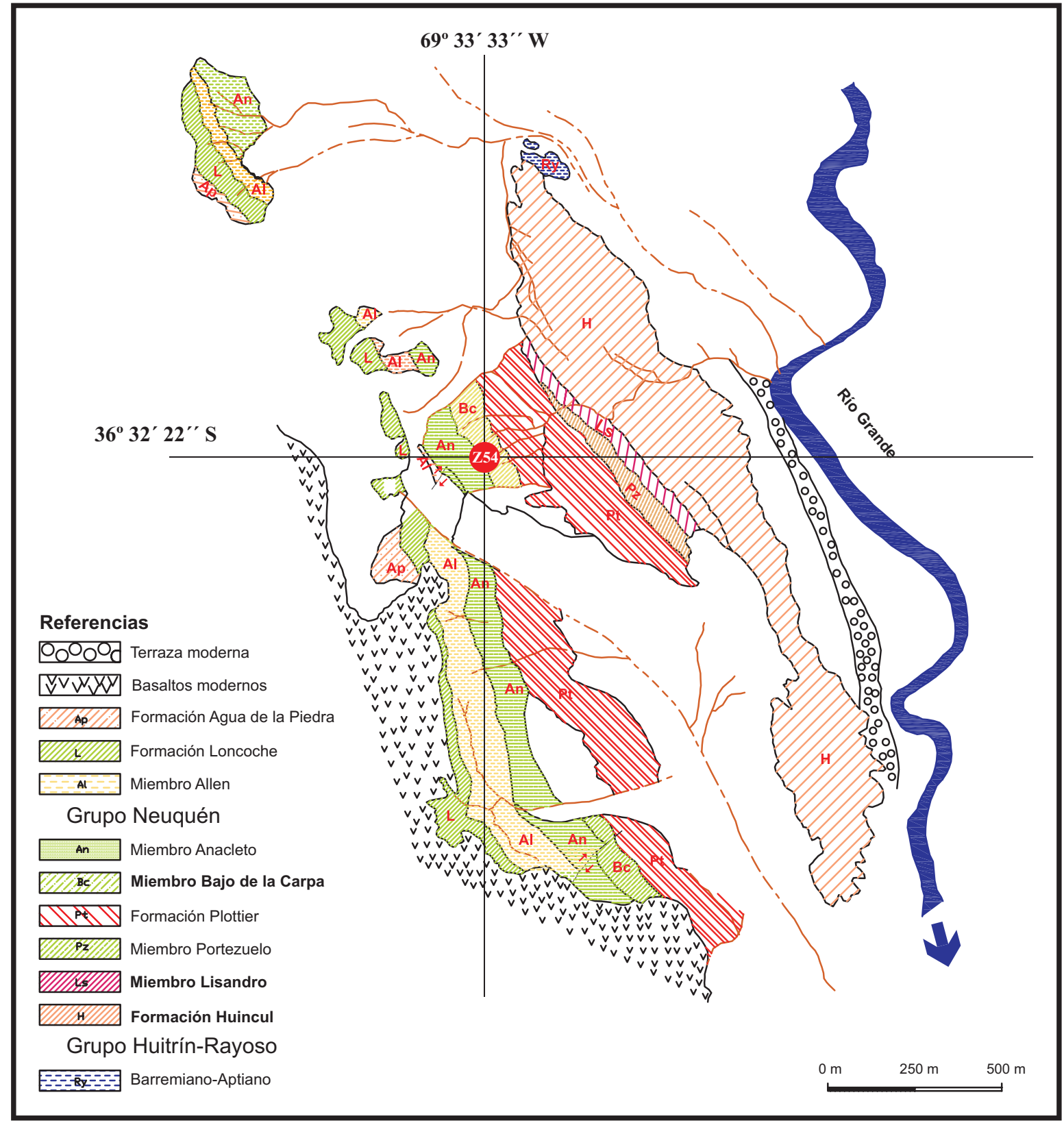

Figura 3. Mapa geológico del afloramiento del Grupo Neuquén en El Zampal, sur de la provincia de Mendoza (modificado de Musacchio 2010). En las referencias incluidas en el mapa se resaltan en negrita las entidades estratigráficas con microfloras estudiadas.

Figure 3. Geological map showing the El Zampal outcrop, Neuquén Group, south of Mendoza province (modified from Musacchio, 2010). The studied stratigraphic units with microflora are highlighted in bold in the references included in the map. 
es un grano triporado presumiblemente endémico en América del Sur, que hasta el momento sólo fue reconocido en la Cuenca Austral, Patagonia argentina (Stough, 1968; Pöthe de Baldis, 1995a), y en distintas cuencas del margen continental brasilero (Regali et al., 1974a; Lima et al., 1986; Azevedo et al., 1987; Regali, 1989). Cretacaeiporites scabratus es un grano periporado intragranulado, descripto originalmente para el Cretácico Superior de África ecuatorial, y presente, además, en distintas cuencas de Brasil. Este taxón se compara con el género actual Thalictrum (Ranunculaceae) y con las Caryophyllaceas y Hamamelidaceas (Herngreen, 1974; Boltenhagen, 1975b). Se menciona, además, la presencia en la asociación de otros palinomorfos conspicuos del Cretácico $\mathrm{Su}$ perior de la Región Ecuatorial como Gabonisporis y Crassitricolporites (Boltenhagen, 1975a; Herngreen et al., 1996).

\section{Microflora de Allen}

En las muestras estudiadas a lo largo del perfil de Lomas Coloradas se advierte un cambio composicional (en cuanto a la participación de grupos de palinomorfos) que sugiere ambientes deposicionales diferentes para esta unidad litológica. En la parte inferior de la entidad (Figura 6, muestras 387-389) predomina el fitoplancton (Botryococcus y phycomata de prasinofitas), con la presencia de helechos heterosporados (Azolla, Grapnelispora); mientras que entre los palinomorfos terrestres la mayor participación corresponde a elementos higrófilos (esporas de pteridofitas, licofitas y briofitas). No se han registrado quistes de dinoflagelados. Esta asociación sugiere la presencia de cuerpos de agua dulce o salobre y condiciones de humedad ambiental.

Hacia la parte superior del perfil los palinomorfos acuáticos van disminuyendo su participación y están ausentes en el tope de la sección. La asociación incluye granos de podocarpáceas dominantes (más del 50\%), como Podocarpidites spp., Phyllocladidites mawsonii y Dacrycarpites australiensis. Estos dos últimos taxa tienen interés biogeográfico porque son formas restringidas a la Región Austral (Herngreen et al., 1996). Por su parte, los taxa actuales relacionados con estos palinomorfos se distribuyen en Australasia (Baldoni \& Askin, 1993; Herngreen et al., 1996).

Los granos de angiospermas están bien representados (30\%), con el polen de probable afinidad proteácea (granos triporados y tricolpoidados) representando cerca del $40 \%$ de este grupo de palinomorfos. La composición de esta asociación se compara con otras similares en latitudes australes caracterizadas por coníferas podocarpáceas dominantes, Proteaceae y Nothofagidites, que representan bosques templado-fríos y húmedos. Sin embargo, la asociación de Allen sugiere condiciones climáticas probablemente más cálidas que las propuestas para los bosques templadohúmedos de la región antártico-australiana. Esto podría estar relacionado con la presencia en la microflora de Allen de algunos taxa compartidos con la región florística paleoecuatorial (Tricesticillus, Gabonisporis, Spinizonocolpites?).

\section{EDAD Y CORRELACIÓN}

\section{Asociación de Confossia vulgaris-Cretacaeiporites scabratus (Santoniano-Campaniano inferior?)}

La especie Confossia vulgaris, que caracteriza la microflora de Bajo de la Carpa, está presente en distintas cuencas marginales de Brasil. Regali (1974b) propuso un rango de distribución

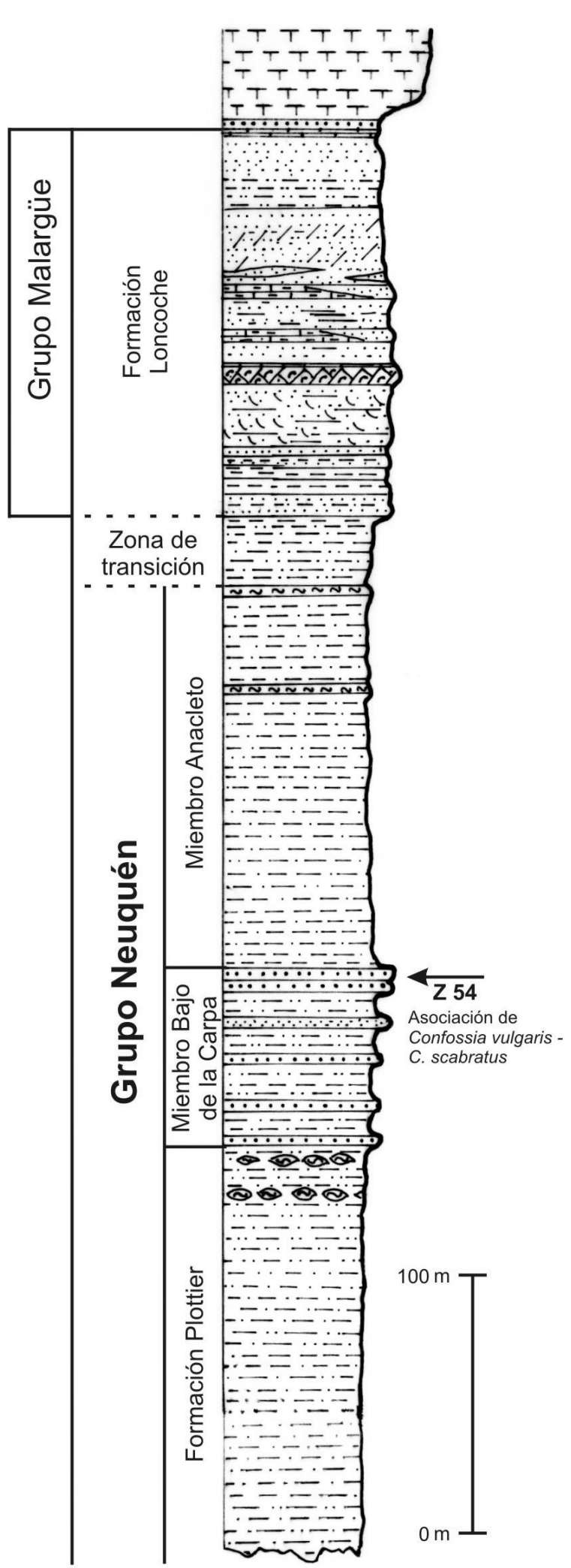

Figura 4. Sección esquemática de la parte superior del Grupo Neuquén en El Zampal (modificado de Uliana \& Musacchio, 1978). La flecha indica el nivel con Confossia vulgaris.

Figure 4. Schematic section of the upper part of the Neuquén Group at El Zampal (modified from Uliana \& Musacchio, 1978). The arrow indicates the level with Confossia vulgaris. 
Coniaciano-Santoniano y base del Campaniano para esta especie, que clasificó como Bahiasporites reticulatus (ver Apéndice 1). Según datos actualizados, el intervalo de distribución de C. vulgaris en Brasil es Santoniano-Campaniano medio (C.C. Lana, com. pers.).

Cretacaeiporites scabratus es una especie común en asociaciones del Cretácico Superior de Brasil y África. En las cuencas brasileras tiene una distribución en el intervalo AlbianoCampaniano superior (C.C. Lana, com. pers.). En África, esta especie tiene un rango comprendido entre el Albiano superior y el Santoniano (Mamhoud \& Mamhoud, 2007).

Classopollis sp. (Figuras 7D-E) es una especie característica en la microflora de Bajo de la Carpa, que incluye granos relativamente grandes (30 a $40 \mu \mathrm{m})$, con estrías conspicuas, un poro desarrollado y pliegues radiales. Formas idénticas a los ejemplares de Bajo de la Carpa han sido reportadas en el Cretácico Superior del Atlántico Sur (McLachlan \& Pieterse, 1978; Ioannides \& Colin, 1977).

Teniendo en cuenta la distribución de los taxones arriba mencionados, y la ausencia en Bajo de la Carpa de polen característico del Campaniano como Tricesticillus y Peninsulapollis, el intervalo cronoestratigráfico más probable para esta asociación es Santoniano-Campaniano inferior?.

\section{Asociación de Tricesticillus tetris-Peninsulapollis gilli (Campaniano-Maastrichtiano)}

Esta asociación de angiospermas incluye entre los taxones con interés bioestratigráfico a Tricesticillus, un taxón común en cuencas marinas del Cretácico Superior brasilero, donde tiene un rango estratigráfico Campaniano medioMaastrichtiano inferior (C.C. Lana, com. pers.). Los granos tricolpoidados como Peninsulapollis gilli (Figuras 10M-N) y los granos tipo Beaupreaidites (Figura 10O), considerados posibles proteáceas, tienen un registro en latitudes australes a partir del Campaniano (Dettmann \& Jarzen, 1988). Entre los granos triporados de probable afinidad proteácea, Lewalanipollis senectus tiene su primer registro en el Santoniano de Australia, y una presencia consistente en la Cuenca Otway durante el Campaniano y el Daniano (Dettmann \& Jarzen, 1996). Tricolpites reticulatus también tiene su primer registro en el Campaniano de latitudes australes. La megaspora Grapnelispora loncochensis es una especie característica en el Campaniano-Maastrichtiano de Argentina (Sepúlveda et al., 1989; Papú, 1997). Las másulas de Azolla (Salviniaceae) también han sido registradas mundialmente a partir del Campaniano (Papú et al., 1988).

Por otra parte, el conjunto sedimentario con la microflora de Allen está situado entre los depósitos continentales infrayacentes de la Formación Anacleto (última entidad del Grupo Neuquén) asignada, al menos en parte, al Campaniano (Musacchio \& Simeoni, 1991) y los sedimentos marinos suprayacentes de la Formación Jagüel. Estos últimos incluyen foraminíferos planctónicos del Maastrichtiano medio (Bertels, 1970). El conjunto de datos previamente expuestos sugiere una edad CampanianoMaastrichtiano para esta asociación.

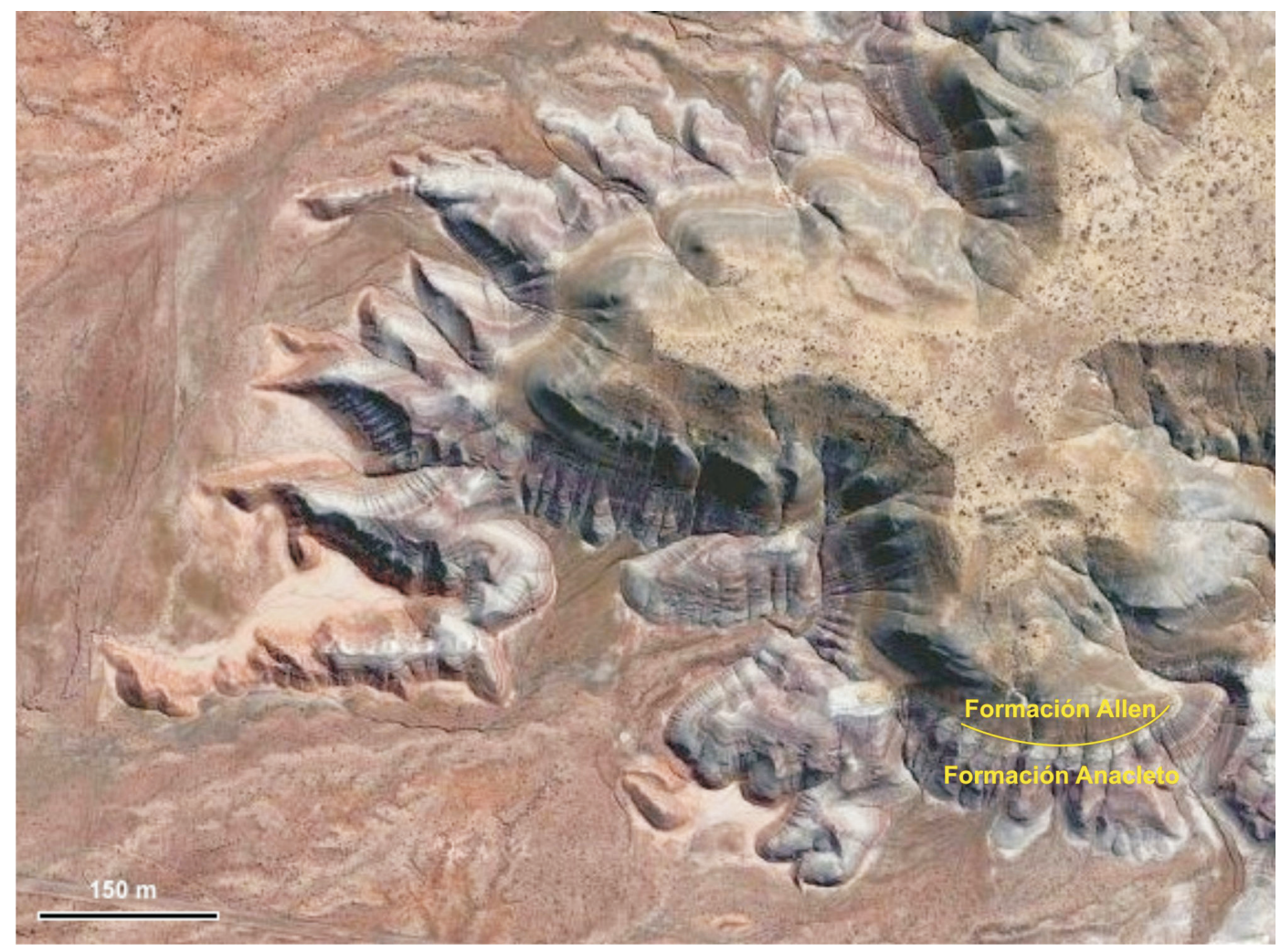

Figura 5. Imagen satelital del afloramiento en la localidad Lomas Coloradas. Se indica el deslinde entre la Formación Allen y la infrayacente Formación Anacleto.

Figure 5. Satellital image of the outcrop at Lomas Coloradas. The boundary between the Allen Formation and the underlying Anacleto Formation is shown. 


\section{SISTEMÁTICA DESCRIPTIVA}

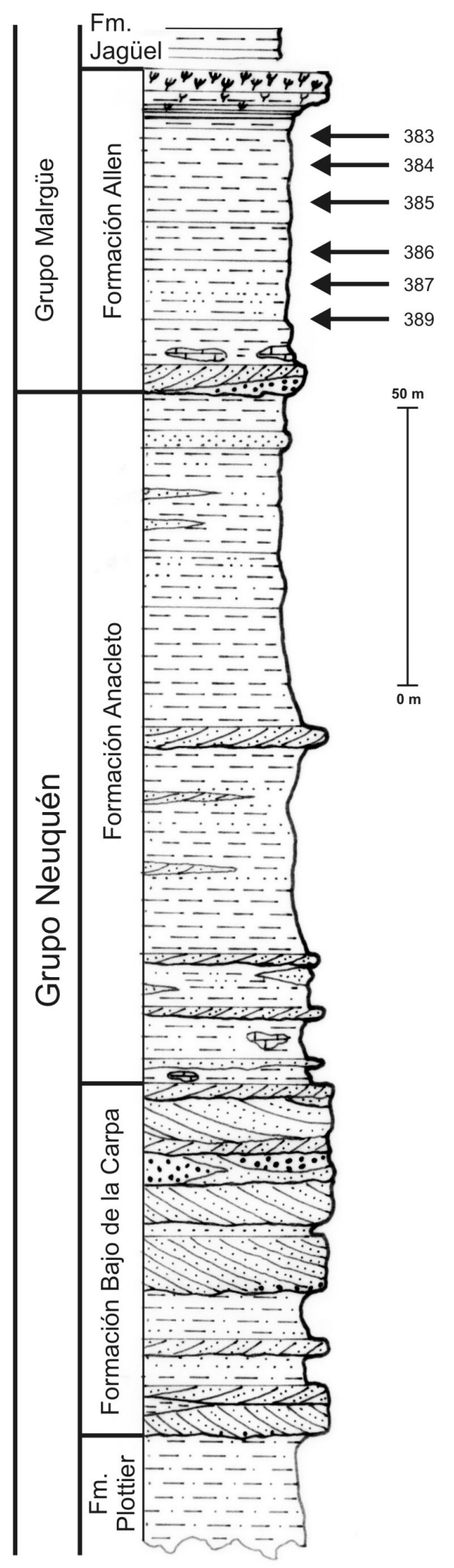

Figura 6. Sección esquemática en Lomas Coloradas con flechas indicando los principales niveles fértiles (modificado de Uliana, 1979).

Figure 6. Schematic section at Lomas Coloradas with arrows showing the main fertile levels (modified from Uliana, 1979).
Anteturma POLLENITES Potonié, 1931

Turma POROSES Naumova emend. Potonié, 1960

Subturma POLYPORINES Naumova emend. Potonié, 1960

Género Tricesticillus Stough, 1968

Especie tipo. Tricesticillus americanus Stough, 1968.

Diagnosis revisada. Polen prolado, pared interna continua, con tres o cuatro bandas meridionales engrosadas, que llevan tres o cuatro poros respectivamente. Las bandas meridionales se unen en las areas polares formando estructuras triradiadas o tetraradiadas.

Revised diagnosis. Prolate pollen, continuous inner wall, with three to four meridional bands, which carry three to four pores respectively. Meridional bands joined in the polar areas forming triradiate or tetraradiate structures.

Consideraciones. Stough (1968) incluyó en el género sólo granos prolados que llevan tres bandas elípticas y tres poros en cada banda. La diagnosis del género Tricesticillus debe ser ampliada sobre la base del nuevo material disponible, para incluir en el mismo taxón granos con cuatro bandas meridionales y cuatro poros en cada una de ellas.

Tricesticillus tetris sp. nov.

(Figuras 11E-L)

Etimología. L. tetris, relacionado con su característica morfología con cuatro bandas longitudinales con cuatro poros cada una. Holotipo. CR.P.CV 383 5/102. Laboratorio de Bioestratigrafía, Facultad de Ciencias Naturales, Universidad Nacional de la Patagonia San Juan Bosco, Comodoro Rivadavia.

Paratipos. CR.P.CV 389 10/94, 10/90.

Material. CR.P.CV 383R 11/89 (ejemplar en vista polar); 383 5/ 102, 21/100; 383A 2.5/92; 383B 12/96; 385A 25/107; 388 20/84; 389A 1/113;389 15/97, 7/100, 10/94, 10/90; 389R1a 13/104, 17/114. Dimensiones. Longitud del grano: $26 \mu \mathrm{m}-30,4 \mu \mathrm{m}$; ancho de la banda meridional: $3 \mu \mathrm{m}-5,6 \mu \mathrm{m}$ (14 ejemplares medidos).

Localidad tipo. Lomas Coloradas, Cuenca Neuquina, Argentina. Distribución estratigráfica. Formación Allen, Grupo Malargüe, Cuenca Neuquina, Argentina.

Diagnosis. Granos de polen prolados. La exina lleva cuatro bandas meridionales engrosadas que se unen en los polos formando una estructura tetraradiada. Cada banda lleva cuatro poros, dos se ubican en la región subpolar de cada hemisferio y los otros dos en posición subecuatorial.

Diagnosis. Prolate pollen grains. Exine with four thickened meridional bands joined at the poles forming a tetraradiate structure. Each band has four pores, two of which are in the subpolar region of each hemisphere and the other two in a subequatorial position.

Descripción. Granos de polen prolados, con un contorno ovalado a subcircular. La exina tiene un espesor de menos de $1 \mu \mathrm{m}$. La pared interna es subgranulosa. Sobre ésta se disponen cuatro bandas meridionales engrosadas que se unen en los polos formando una estructura tetraradiada (Figura 11F). Cada banda lleva cuatro poros, dos de ellos se ubican en la región subpolar de cada hemisferio y los otros dos en posición subecuatorial (Figuras 11G,J). Los poros, de contorno circular a oval, se disponen en un área adelgazada central, a lo largo de cada banda meridional, de ancho variable (hasta $3.5 \mu \mathrm{m}$ en el ejemplar de la Figura 11J). Las bandas pueden presentar un borde festoneado, como se observa en el espécimen ilustrado en la Figura 11J. 

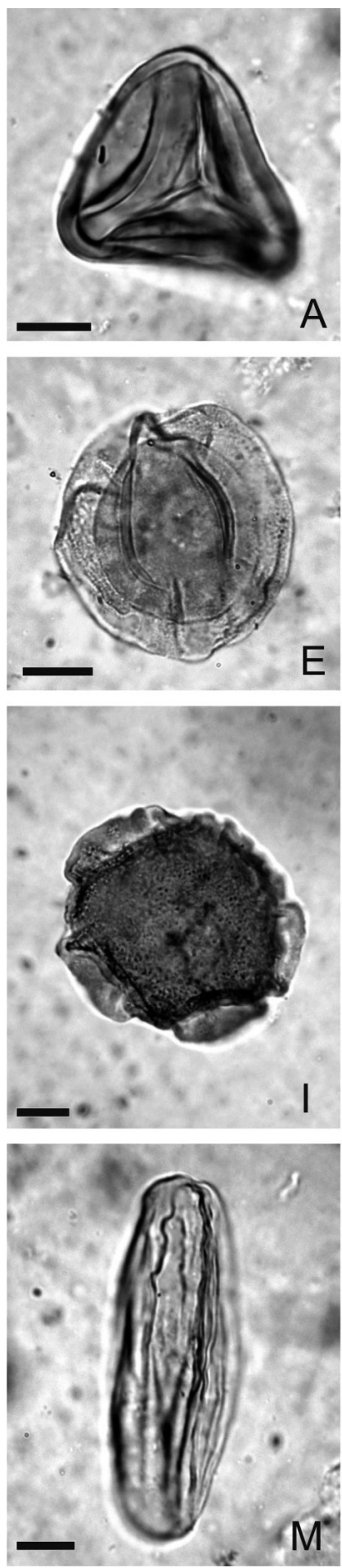
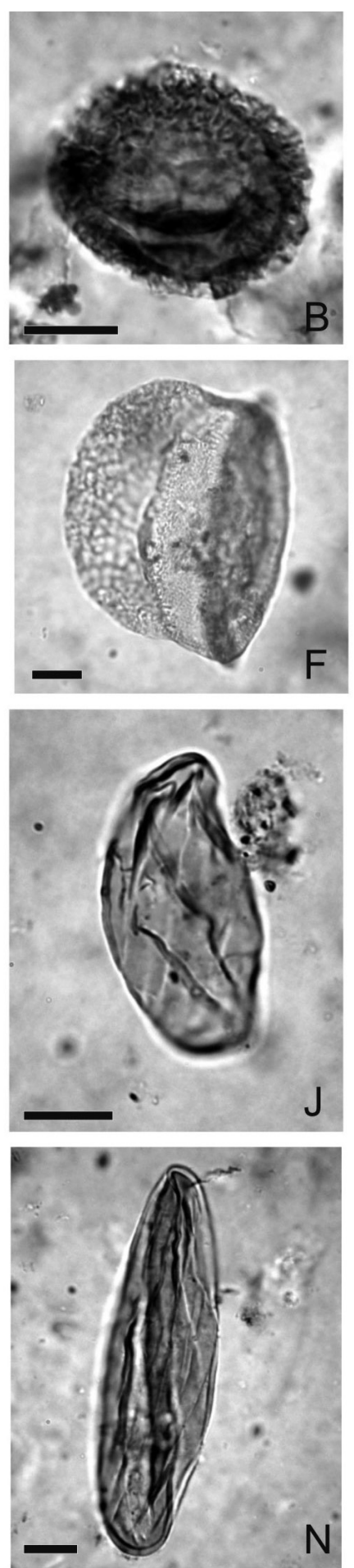
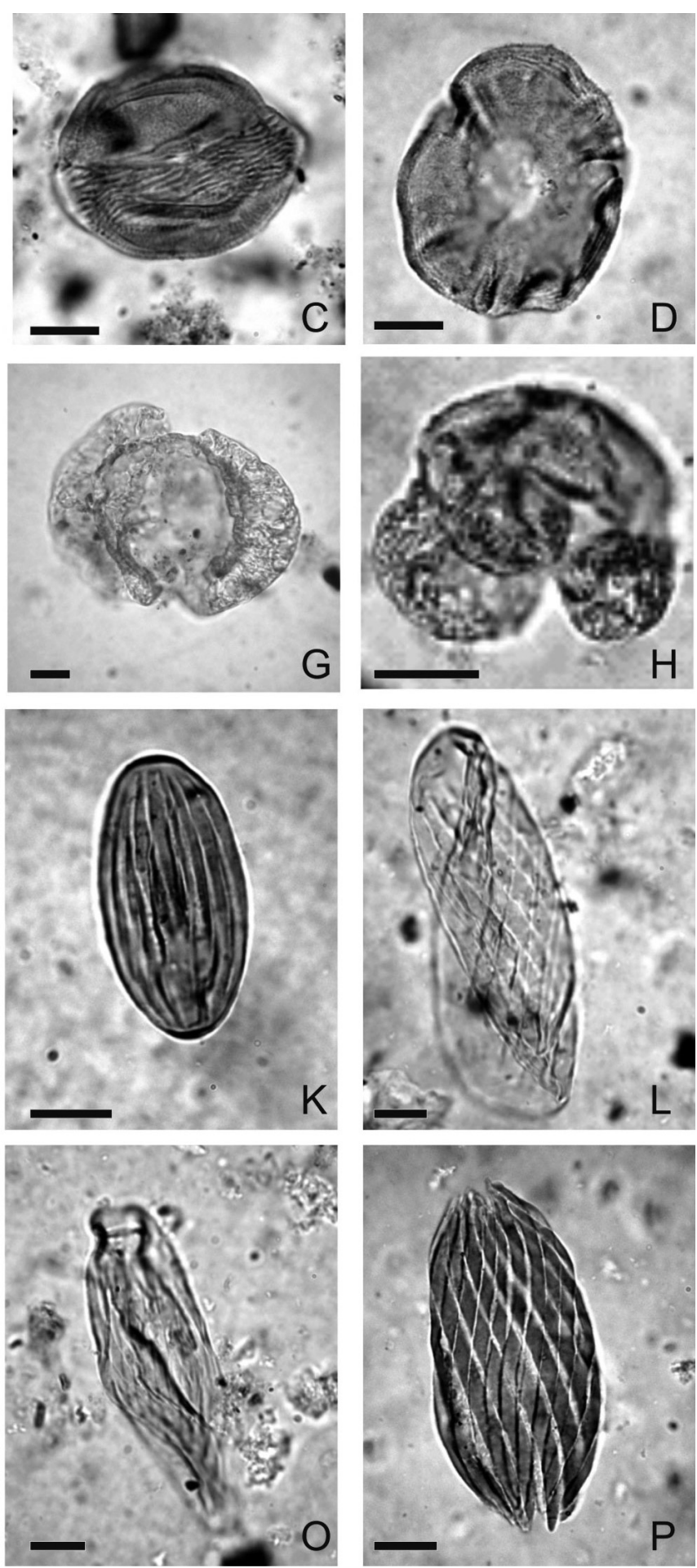

Figura 7. Asociación de Confossia vulgaris-Cretacaeiporites scabratus (Santoniano-Campaniano inferior?). Esporas y granos de polen de gimnospermas. En paréntesis están los números de los preparados y las coordenadas en el microscopio citado. A, Gleicheniidites senonicus (CR.P.CV 54C 8.5/100); B, Gabonisporis vigourouxii (CR.P.CV 543P 13/94); C, Classopollis classoides (CR.P.CV 543P 5/39); D, Classopollis sp. (CR.P.CV 54 C 18/100); E, Classopollis sp. (CR.P.CV 54P 11/98); F, Podocarpidites cf. marwicki (CR.P.CV 5430 6/89); G, Podocarpidites rugulosus (CR.P.CV 543L 18/95); H, Microcachryidites antarcticus (CR.P.CV 543L 16.5/96); I, Callialasporites trilobatus (CR.P.CV 54i 12/100); J, Equisetosporites cf. cancellatus (CR.P.CV 543L 17/97); K, Equisetosporites multicostatus (CR.P.CV 543P 13/94); L, Equisetosporites cf. albertensis (CR.P.CV 543L 14/100); M, Singhia sp. (CR.P.CV 54C 5/104); N, Equisetosporites sp. (CR.P.CV 54C 14/105); O, Steevesipollenites sp. (CR.P.CV 54C 18.5/90); P, Gnetaceaepollenites jansonii (CR.P.CV 541 9.5/97). Escalas $=10 \mu \mathrm{m}$.

Figure 7. Confossia vulgaris-Cretacaeiporites scabratus Assemblage (Santonian-?lower Campanian). Spores and gymnosperm pollen grains. In parenthesis slide numbers and coordinates in the cited microscope. Scale bars $=10 \mu \mathrm{m}$. 

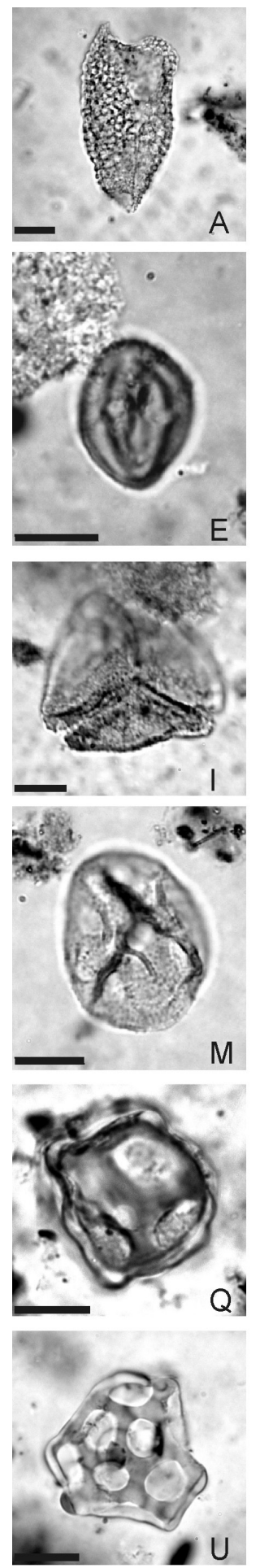
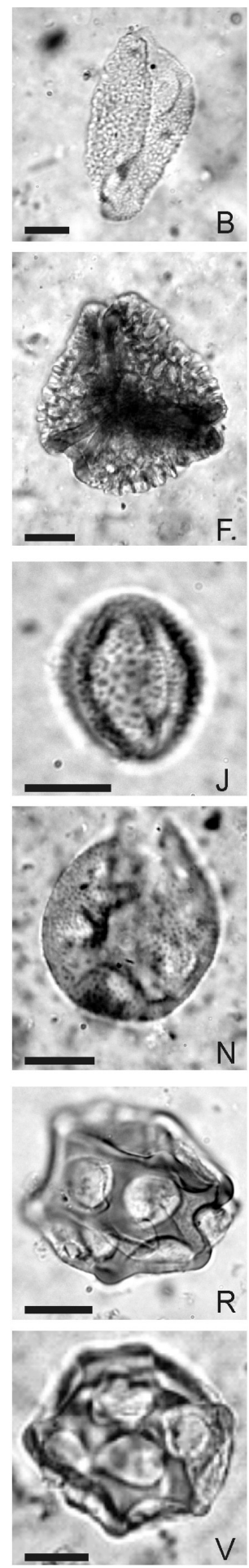
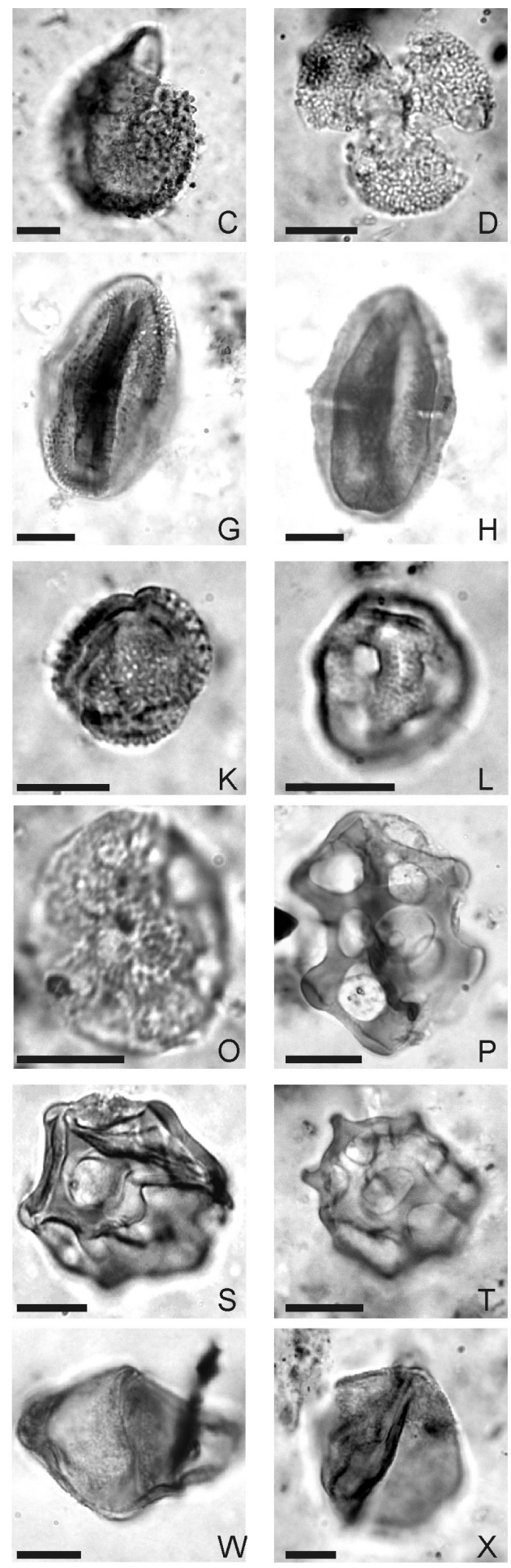

Figura 8. Asociación de Confossia vulgaris-Cretacaeiporites scabratus (Santoniano-Campaniano inferior?). Granos de polen de angiospermas. En paréntesis están los números de los preparados y las coordenadas en el microscopio citado. A, Liliacidites cf. kaitangataensis (CR.P.CV 54 3N 8/109); B, Liliacidites sp. (CR.P.CV 54C 4/94); C, Racemonocolpites? sp. (CR.P.CV 543N 6/108); D, Tricolpites sp. (CR.P.CV 54j 97/111); E, Psilatricolporites sp. (CR.P.CV 54C 20/90); F, Cupanieidites? sp. (CR.P.CV 543A 3/81.5); G, Crassitricolporites sp. (CR.P.CV 54C 8.5/102); H Crassitricolporites sp. (otro foco del especimen ilustrado en G); I, Syncolporites? sp. (CR.P.CV 543N 8/109); J, Dryadopollis sp. (CR.P.CV 543N 4/103) vista ecuatorial; K, Dryadopollis sp. (CR.P.CV 5430 5/90) vista oblicua; L, Cretacaeiporites cf. scabratus (CR.P.CV 54h 7/101); M, Cretacaeiporites scabratus (CR.P.CV 543M 15/108); N, Cretacaeiporites scabratus (CR.P.CV 543B 12/95); O, Cretacaeiporites scabratus (CR.P.CV 543N 12/100); P, Confossia vulgaris (CR.P.CV 540 20/95); Q, Confossia vulgaris (CR.P.CV 543P 5/90); R, Confossia vulgaris (CR.P.CV 543L 17/98); S, Confossia vulgaris (CR.P.CV 543L 17/98); T, Confossia vulgaris (CR.P.CV 54P 3.5/90); U, Confossia vulgaris (CR.P.CV 543L 10/91); V, Confossia vulgaris (CR.P.CV 54EZ3O 17/90); W, Propyllipollis? sp. (CR.P.CV 54C 8/102) vista lateral; X, Proteacidites? sp. (CR.P.CV 543L 8/93) vista lateral. Escalas $=10 \mu \mathrm{m}$.

Figure 8. Confossia vulgaris-Cretacaeiporites scabratus Assemblage (Santonian-?lower Campanian). Angiosperm pollen grains. In parenthesis slide numbers and coordinates in the cited microscope. Scale bars $=10 \mu \mathrm{m}$. 

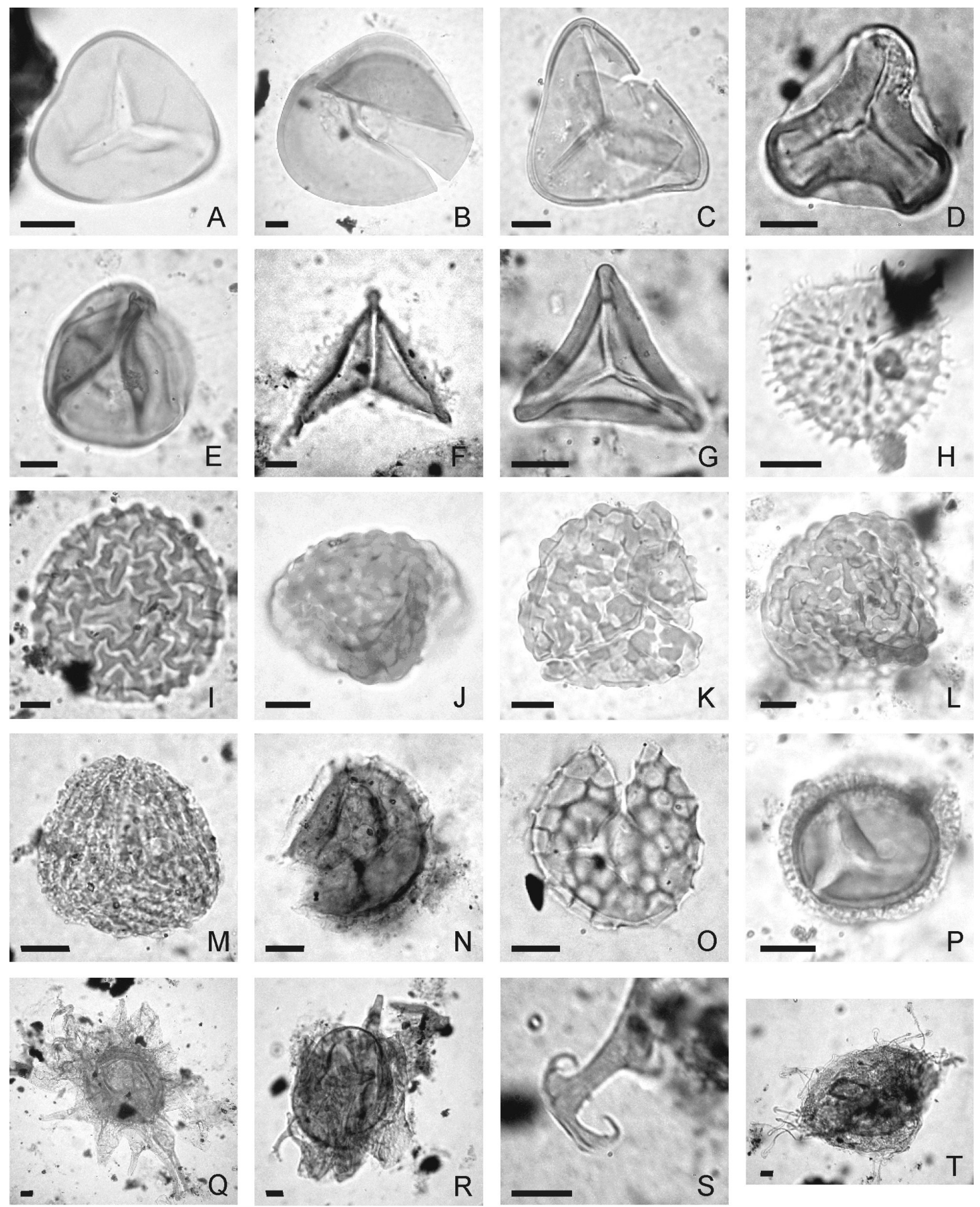

Figura 9. Asociación de Tricesticillus tetris-Peninsulapollis gilli (Campaniano-Maastrichtiano). Esporas. En paréntesis están los números de los preparados y las coordenadas en el microscopio citado. A, Cyathidites minor (CR.P.CV 389A 4/84); B, Cyathidites patagonicus (CR.P.CV 389A 22/107); C, Cyathidites sp. (CR.P.CV 389A 19/103); D, Dictyophyllidites sp. (CR.P.CV 389A 3/110); E, Biretisporites sp. (CR.P.CV 389A 18/99); F, Clavifera triplex (CR.P.CV 383 19/110); G, Gleicheniidites senonicus (CR.P.CV 389R1A 20/102); H, Ceratosporites equalis (CR.P.CV 389A 7/106); I, Camarozonosporites sp. (CR.P.CV 389A 4/84); J, Klukisporites sp. (CR.P.CV 389 R1a 8/105); K, Hamulatisporis sp. cf. Rugulatisporites rionegrensis (CR.P.CV 389A 23/85); L, Hamulatisporis sp. (CR.P.CV 384 8.5/105); M, Cicatricosisporites sp. (CR.P.CV 387A 12/88); N, Triporoletes reticulatus (CR.P.CV 383B 3/93); O, Retitriletes austroclavadites (CR.P.CV 389A 3/93); P, Gabonisporis sp. (CR.P.CV 389R1a 20/104); Q, Grapnelispora loncochensis (CR.P.CV 389 20/92); R, Grapnelispora loncochensis (CR.P.CV 389 6/112); S, Grapnelispora loncochensis (CR.P.CV 389 6/112) detalle del proceso; T, másula de Azolla sp. (CR.P.CV 389A 4/84). Escalas $=10 \mu \mathrm{m}$.

Figure 9. Tricesticillus tetris-Peninsulapollis gilli Asemblage (Campanian-Maastrichtian). Spores. In parenthesis slide numbers and coordinates in the cited microscope. Scale bars $=10 \mu \mathrm{m}$. 

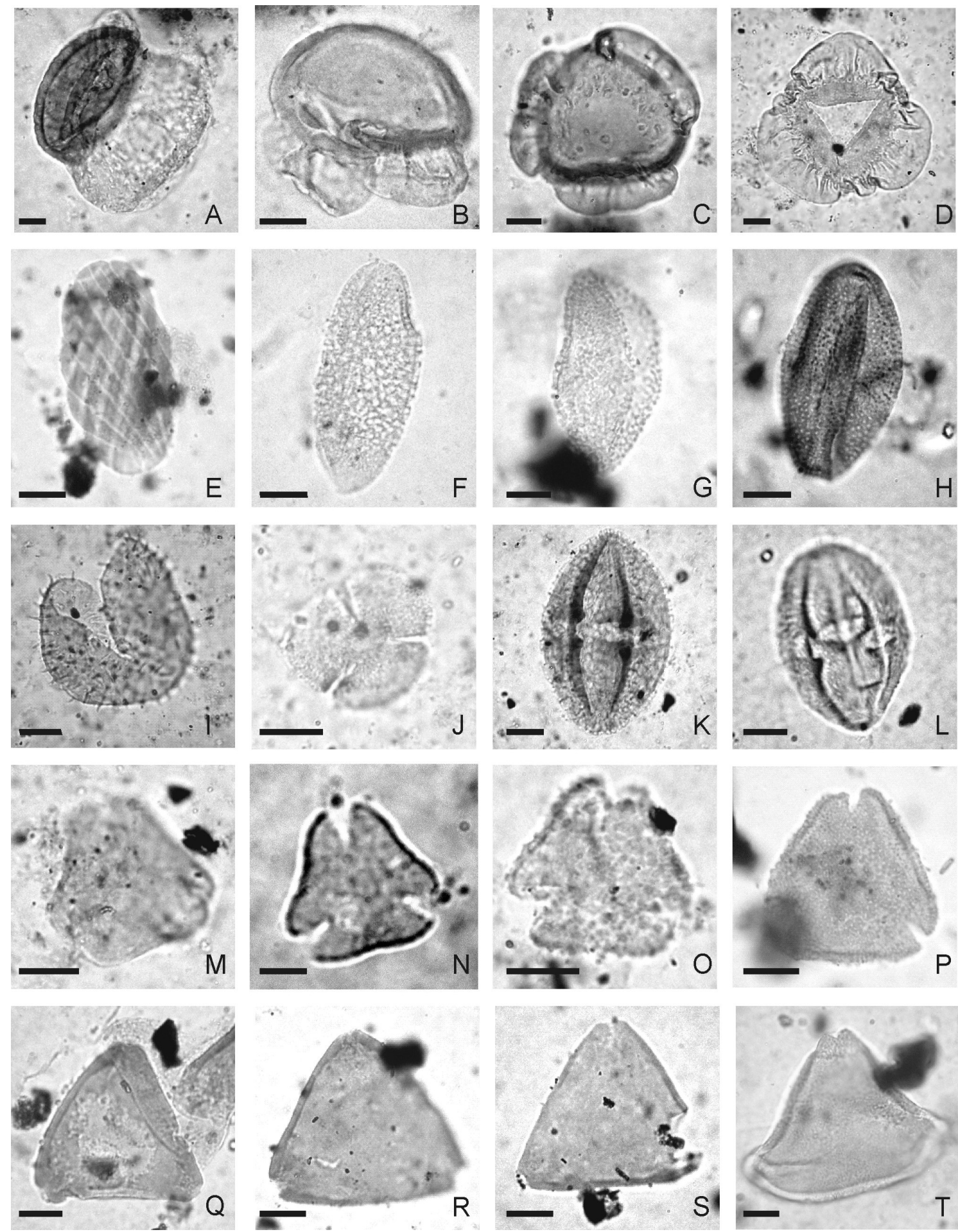

Figura 10. Asociación de Tricesticillus tetris-Peninsulapollis gilli (Campaniano-Maastrichtiano). Granos de polen de gimnospermas y angiospermas. En paréntesis están los números de los preparados y las coordenadas en el microscopio citado. A, Podocarpidites cf. auriculatus (CR.P.CV 3831 24/110); B, Phyllocladidites mawsonii (CR.P.CV 3831 14/85); C, Callialasporites trilobatus (CR.P.CV 38512 ( 95); D, Dacrycarpites australiensis (CR.P.CV 383A 14/91); E, Gnetaceaepollenites jansonii (CR.P.CV 387A 24/87); F, Liliacidites kaitangataensis (CR.P.CV 3831 5/89); G, Clavamonocolpites sp. (CR.P.CV 389A 3/104); H, Fraxinoipollenites sp. (CR.P.CV 389 6/94); I, Spinizonocolpites sp. (CR.P.CV 383A 5/108.5); J, Tricolpites cf. reticulatus (CR.P.CV 383A 15/90); K, Retitricolporites sp. (CR.P.CV 383A 19/87); L, Striatricolporites cf. gamerroi (CR.P.CV 3831 11/108); M, Peninsulapollis gilli (CR.P.CV 383R 13/98); N, Peninsulapollis gilli (CR.P.CV 383B 10/97); O, cf. Beaupreaidites sp. (CR.P.CV 383 2.5/97); P, Peninsulapollis sp. (CR.P.CV 387A 12/105); Q, Lewalanipollis cf. senectus (CR.P.CV 383B 7/90) restos de una tétrade; R, Lewalanipollis cf. senectus (CR.P.CV 385B 2/93); S, Lewalanipollis cf. senectus (CR.P.CV 384A 15/107); T, Propyllipollis sp. (CR.P.CV 389A 2.5/103). Escalas = $10 \mu \mathrm{m}$.

Figure 10. Tricesticillus tetris-Peninsulapollis gilli Assemblage (Campanian-Maastrichtian). Gymnosperm and angiosperm pollen grains. In parenthesis slide numbers and coordinates in the cited microscope. Scale bars $=10 \mu \mathrm{m}$. 

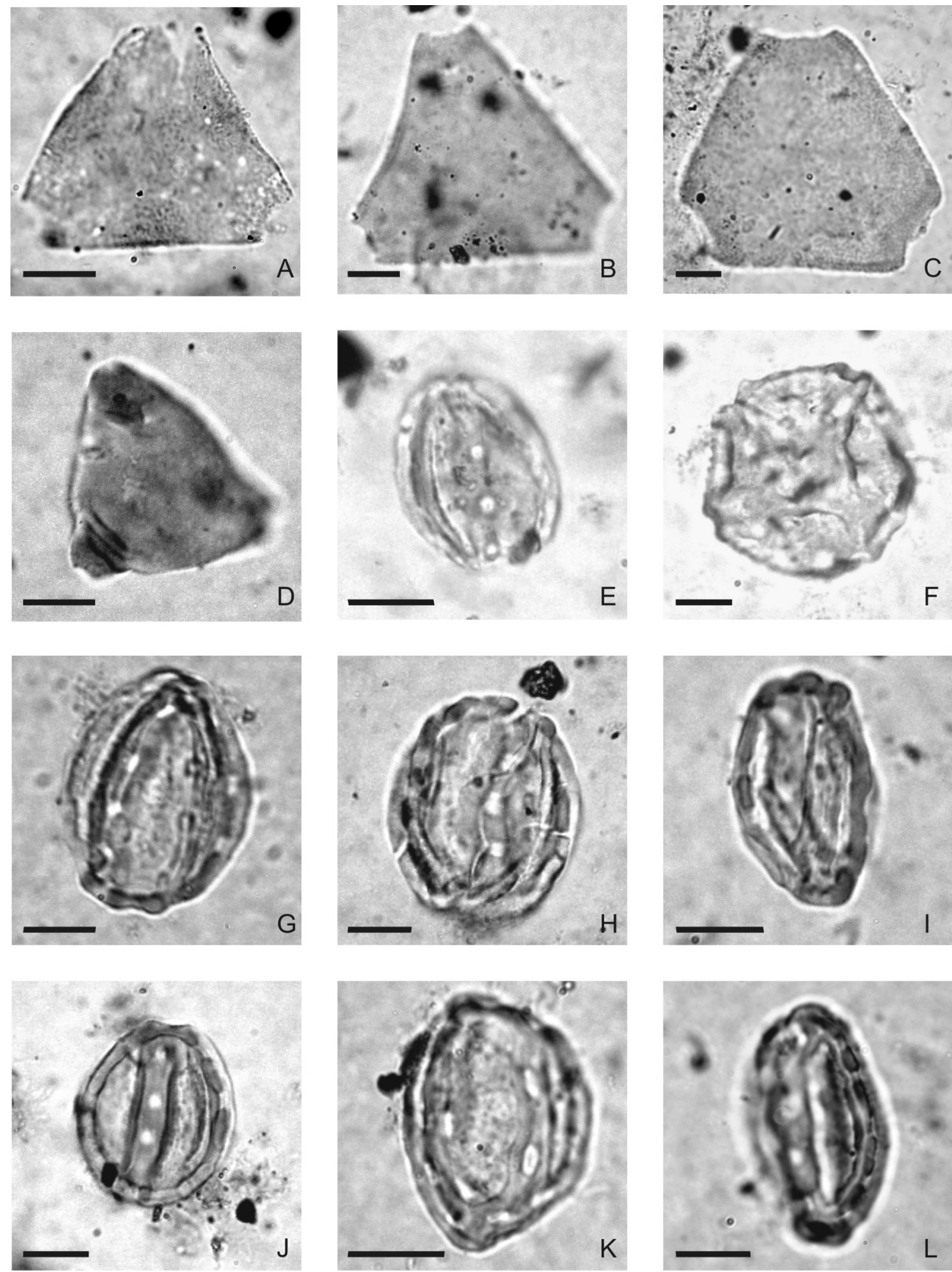

Figura 11. Asociación de Tricesticillus tetris-Peninsulapollis gilli (Campaniano-Maastrichtiano). Granos de polen de angiospermas. En paréntesis están los números de los preparados y las coordenadas en el microscopio citado. A, Proteacidites cf. tenuiexinus (CR.P.CV 389A 2.5/103); B, Proteacidites? sp. (Cr.P.CV 397 13/95); C, Proteacidites sp. (CR.P.CV 3831 23/93); D, cf. Propyllipollis lateflexus (CR.P.CV 389A 10/113.5); E, Tricesticillus tetris (CR.P.CV 389 R1a 13/104); F, Tricesticillus tetris (CR.P.CV 383R 11/89) vista polar; G, Tricesticillus tetris (CR.P.CV 383 5/102); H, Tricesticillus tetris (CR.P.CV 383B 12/96); I, Tricesticillus tetris (CR.P.CV 389 10/90); J, Tricesticillus tetris (CR.P.CV 389 7/100); K, Tricesticillus tetris (CR.P.CV 389 10/94); L, Tricesticillus tetris (CR.P.CV 389A 1/113). Escalas $=10 \mu \mathrm{m}$.

Figure 11. Tricesticillus tetris-Peninsulapollis gilli Assemblage (Campanian-Maastrichtian). Angiosperm pollen grains. In parenthesis slide numbers and coordinates in the cited microscope. Scale bars $=10 \mu \mathrm{m}$. 
Comparaciones. La especie tipo para el género fue reconocida originalmente en la Formación Mata Amarilla de la Cuenca Austral argentina (Stough, 1968). Esta especie incluye tres bandas meridionales engrosadas en la exina. Las mismas llevan tres poros en cada una de ellas (dos en posición subpolar y uno ecuatorial). Las bandas se interconectan en las áreas polares formando una estructura triradiada.

Formas similares a las reconocidas en Allen han sido registradas en el Cretácico Superior de distintas cuencas del margen continental brasilero (C.C. Lana \& M. Arai, com. pers.). Se sugieren estudios complementarios sobre la distribución estratigráfica de estos palinomorfos para interpretar estas diferencias observadas.

\section{DISCUSIÓN}

Las dos microfloras del Cretácico Superior ahora presentadas con diferencias composicionales y elementos diagnósticos propios de cada asociación, sugieren cambios en las relaciones paleobiogeográficas. En efecto, la marcada presencia en la parte superior del Grupo Neuquén de los elementos paleoecuatoriales anteriormente mencionados (Confossia vulgaris, Cretacaeiporites scabratus, Crassitricolporites sp., Classopollis sp., Gabonisporis vigourouxii), junto a otros taxa conspicuos de la Región Paleoflorística Austral, como Microcachryidites antarcticus y Liliacidites cf. kaitangataensis, sugieren que las condiciones transicionales que se habían propuesto previamente para las asociaciones palinológicas del Aptiano y el Cenomaniano de la Cuenca Neuquina (Vallati, 2002b) se mantienen en el Santoniano.

Por otra parte, la presencia en la asociación de Bajo de la Carpa de granos triporados de posible afinidad proteacea, como Propyllipollis? sp. y Proteacidites? sp. (Figuras 8W$\mathrm{X}$ ), parece de interés en los estudios sobre evolución y dispersión de las Proteaceae (ver Askin \& Baldoni, 1998), teniendo en cuenta que éste constituye el primer registro para Argentina de polen de esta familia en sedimentos con una probable antigüedad pre-campaniana.

En la Formación San Carlos del Grupo Baurú, Brasil, y en particular en el Miembro Fazenda Nossa Senhora de Fátima, fue reportada una asociación con Confossia vulgaris asignada al intervalo Coniaciano-Santoniano (Castro et al., 2002), la que se compara bien con la microflora de Bajo de la Carpa. Además de Confossia vulgaris otros taxones compartidos entre las mencionadas asociaciones son Gabonisporis vigourouxii, Gnetaceapollenites jansonii, Classopollis, Cretacaeiporites (Lima et al., 1986; Castro et al., 2002). Las relaciones entre los depósitos pre-campanianos del Grupo Neuquén en la Cuenca Neuquina y el mencionado Grupo Baurú fueron previamente sugeridas en base a la similitud de las asociaciones de microfósiles calcáreos en ambas entidades (Musacchio et al., 2002; Castro et al., 2002). Más recientemente, Candeiro \& Martinelli (2006) sugieren también correspondencias entre las faunas de mesoeucrocodylia de las mencionadas entidades brasilera y neuquina.

En la Cuenca Austral, en el sudoeste de Argentina también hay registro de asociaciones comparables a la microflora de Bajo de la Carpa. Stough (1968) reconoce por primera vez la especie Confossia vulgaris en sedimentos del Cretácico
Superior próximos al Lago Argentino. Asimismo, Pöthe de Baldis (1995a) reporta una asociación con Confossia vulgaris en la Formación Río Guanaco, en un perfil comprendido entre el Lago Viedma y el Lago San Martín. Una asociación de dinoflagelados provenientes de facies marinas de esta misma unidad litoestratigráfica fue asignada al Santoniano (Pöthe de Baldis, 1995b).

La microflora de Allen, en Lomas Coloradas, y en particular la asociación presente en los niveles superiores de la unidad, con predominio de polen sacado de podocarpáceas y con granos triporados y tricolpoidados es, en parte, comparable en su composición a las floras del Cretácico Superior de latitudes australes (Dettmann \& Thomson, 1987; Dettmann \& Jarzen, 1988, 1996; Askin, 1992; Herngreen et al., 1996). Sin embargo, el polen de Nothofagus (Nothofagidites) no está presente en la Formación Allen, y hace su primera aparición en las asociaciones patagónicas maastrichtianas de la Formación Jagüel (Romero, 1973).

En Argentina, varias microfloras maastrichtianas en las provincias de Chubut, Río Negro y Mendoza incluyen elementos comunes con la presente microflora (Baldoni \& Askin, 1993; Baldoni, 1991; Papú, 1989, 1990, 2002). Asimismo, Papú \& Sepúlveda (1995) reportaron una asociación de edad campaniana-maastrichtiana en la Formación Los Alamitos, provincia de Río Negro, que se compara bien con la asociación de Allen. La correspondencia entre ambas unidades también se extiende a la fauna de vertebrados (ver Rougier et al., 2009).

La microflora de Allen incluye algunos taxa compartidos con la Región Paleoecuatorial (Gabonisporis, Tricesticillus, Spinizonocolpites), pero la afinidad de esta asociación con la región antártico-australiana es mayor que la evidenciada por la microflora de Bajo de la Carpa, en el Grupo Neuquén.

\section{CONCLUSIONES}

Se dan a conocer dos nuevas asociaciones palinológicas con angiospermas en el Cretácico Superior de la Cuenca Neuquina: la asociación de Confossia vulgarisCretacaeiporites scabratus y la asociación de Tricesticillus tetris-Peninsulapollis gilli.

La asociación de angiospermas de la entidad estratigráfica Bajo de la Carpa está caracterizada por la presencia de los taxones Confossia vulgaris y Cretacaeiporites scabratus, bien representados en el Cretácico Superior de Gondwana septentrional. La distribución estratigráfica conocida de estas especies sugiere una edad comprendida en el intervalo Santoniano-Campaniano inferior?. La presencia de Confossia, Cretacaeiporites y otros taxones compartidos con la Región Paleoflorística Ecuatorial (Herngreen et al., 1996) junto a palinomorfos conspicuos de la Región Austral, sugiere condiciones transicionales para la microflora de Bajo de la Carpa, estudiada en El Zampal.

Por otra parte, la Formación Allen, en la localidad de Lomas Coloradas, incluye una asociación de angiospermas caracterizada por taxones endémicos de América del Sur (Tricesticillus) y palinomorfos característicos de Gondwana austral, como Peninsulapollis gilli y granos triporados, de probable afinidad con las proteáceas. En esta microflora se reconoce la especie nueva Tricesticillus tetris. Teniendo en cuenta la asociación palinológica y la presencia de foraminíferos planctónicos del 
Maastrichtiano medio en la suprayacente Formación Jagüel, se sugiere para esta entidad una edad comprendida en el intervalo Campaniano-Maastrichtiano. La composición de la palinoflora de Allen es, en parte, comparable a otras microfloras conocidas de Gondwana austral.

Los cambios paleoecológicos, biogeográficos y cronológicos que parecen existir entre las microfloras de las entidades Bajo de la Carpa y Allen deberán testarse en futuros estudios palinológicos en la Formación Anacleto, con el objeto de aproximar el deslinde bioestratigráfico que se detecta ahora entre las dos primeras.

\section{AGRADECIMIENTOS}

Agradezco E. Musacchio por la lectura crítica del manuscrito y su apreciado asesoramiento en distintos tópicos de esta contribución. Asimismo, agradezco a C.C. Lana y E.P. Ferreira, quienes como árbitros, realizaron sugerencias y aportaron comentarios, que mejoraron esta contribución. Este artículo es una contribución al XII SBPP (Florianópolis, 0205 de noviembre de 2008).

\section{REFERENCIAS}

Archangelsky, S.; Baldoni, A.; Gamerro, J.C. \& Seiler, J. 1983. Palinología estratigráfica del Cretácico de Argentina austral II. Descripciones sistemáticas. Ameghiniana, 20(3-4):199-226.

Archangelsky, S.; Baldoni, A.; Gamerro, J.C. \& Seiler, J. 1984. Palinología estratigráfica del Cretácico de Argentina austral III. Distribución de las especies y conclusiones. Ameghiniana, 21(1):15-33

Archangelsky, S. \& Gamerro, J.C. 1967. Spore and pollen types of the Lower Cretaceous in Patagonia (Argentina). Review of Palaeobotany and Palynology, 1:211-217.

Askin, R.A. 1992. Late Cretaceous-Early Tertiary antarctic outcrop evidence for past vegetation and climates. Antarctic Research Series, 56:61-73.

Askin, R.A. \& Baldoni, A. 1998. The Santonian through Paleogene record of Proteaceae in the Southern South America-Antarctic Peninsula Region. Australian Systematic Botany, 11:379-390.

Azevedo, R.L.M.; Gomide, J.; Viviers, M.C. \& Hashimoto, A.T. 1987. Bioestratigrafia do Cretáceo marinho da Bacia de Campos, Brasil. Revista Brasileira de Geociências, 17(2):147-153.

Baldoni, A.M. 1991. Estudio palinológico de la Localidad El Cain (Cretácico Superior), Provincia de Río Negro, Argentina, y sus relaciones con otros terrenos supracretácicos de Argentina. In: CONGRESO GEOLÓGICO CHILENO, 6, 1991. Resúmenes ampliados, Viña del Mar, p. 84-86.

Baldoni, A.M. \& Askin, R.A. 1993. Palynology of the lower Lefipan Formation (Upper Cretaceous) of Barranca de los Perros, Chubut Province, Argentina. Part II. Angiosperm pollen and discussion. Palynology, 17:241-264.

Bertels, A. 1970. Los foraminíferos planctónicos de la cuenca Cretácico-Terciaria en Patagonia septentrional (Argentina), con consideraciones sobre la estratigrafía de Fortín General Roca. Ameghiniana, 7:1-56.

Boltenhagen, E. 1975a. Quelques spores du Crétacé Supérieur du Gabon. Revue de Micropaléontologie, 18(2):69-80.

Boltenhagen, E. 1975b. Pollen périporé du Crétacé Supérieur du Gabon. Revue de Micropaléontologie, 17(4):164-170.

Candeiro, C.R.A. \& Martinelli, A.G. 2006. A review of paleogeographical and chronostratigraphical distribution of mesoeucrocodylian species from the Upper Cretaceous beds from the Bauru (Brazil) and Neuquén (Argentina) groups,
Southern South America. Journal of South American Earth Sciences, 22:116-129.

Castro, J.C.; Dias-Brito, D.; Arai, M.; Rodríguez, R. \& Musacchio, E.A. 2002. Formação São Carlos: uma nova unidade para o Grupo Bauru (Cretáceo continental do Brasil). In: SIMPOSIO SOBRE EL CRETÁCICO DE AMÉRICA DEL SUR, 2, 2002. Resumos expandidos, São Pedro, UNESP, p. 351-358.

Cazau, L. \& Uliana, M.A. 1973. El Cretácico Superior en la Cuenca Neuquina. Buenos Aires. In: CONGRESO GEOLÓGICO ARGENTINO, 5, 1973. Actas, Buenos Aires, p. 131-163.

Dettmann, M.E. \& Jarzen, D.M. 1988. Angiosperm pollen from uppermost Cretaceous strata of southeastern Australia and the Antarctic Peninsula. Association of Australasian Palaeontologists, Memoir, 5:217-237.

Dettmann, M.E. \& Jarzen, D.M. 1996. Pollen of proteceous-type from latest Cretaceous sediments, southeastern Australia. Alcheringa, 20(2):103-160.

Dettmann, M.E. \& Thomson, M.R.A. 1987. Cretaceous palynomorphs from the James Ross Island Area, Antarctica: a pilot study. British Antarctic Survey Bulletin, 77:13-59.

Gerth H. 1925. Contribuciones a la estratigrafía y paleontología de los Andes argentinos I: estratigrafía y distribución de los sedimentos mesozoicos en los Andes argentinos. Actas de la Academia Nacional de Ciencias de Córdoba, 9(1):7-55.

Herngreen, G.F.W. 1974. Palynology of Albian-Cenomanian strata of borehole 1-QS-1-MA, State of Maranhão, Brazil. Pollen et Spores, 15(3-4):514-555.

Herngreen, G.F.W.; Kedves, M.; Rovnina, L.V. \& Smirnova, S.B 1996. Cretaceous palynofloral provinces: a review. In: J. Jansonius \& D.C. McGregor (eds.) Palynology: principles and applications, American Association of Stratigraphic Palynologists Foundation, 3:1157-1188.

Ioannides, N.S. \& Colin, J.P. 1977. Palynology at sites 358, 356, 355, DSDP Leg 39, Southwestern Atlantic Ocean. Initial Reports of the Deep Sea Drilling Project, 39:885-897.

Leanza, H.A.; Apesteguía, S.; Novas, F.E. \& de la Fuente, M.S. 2004. Cretaceous terrestrial beds from the Neuquén Basin (Argentina) and their tetrapod assemblages. Cretaceous Research, 25(1):61-87.

Lima, M.R.; Mezzalira S.; Dino, R. \& Saad A.R. 1986. Descoberta de microflora em sedimentos do Grupo Bauru, Cretáceo do Estado de São Paulo. Revista do Instituto Geológico, 7(1/2):5-9.

Mahmoud, M.S. \& Mahmoud, A.E. 2007. Palynology of some Cretaceous mudstones from southeast Aswan, Egypt: significance to regional stratigraphy. Journal of African Earth Sciences, 47:1-8.

McLachlan, I.R. \& Pieterse, E. 1978. Preliminary palynological results: site 361, leg 40, Deep Sea Drilling Project. Initial Reports of the Deep Sea Drilling Project, 40:857-881.

Musacchio, E.A. 1973. Charophytas y ostrácodos no marinos del Grupo Neuquén (Cretácico Superior) en algunos afloramientos de las Provincias de Río Negro y Neuquén en la República Argentina. Revista del Museo de La Plata (Nueva Serie), Sección Paleontología, 8:1-33.

Musacchio, E.A. 1989. Biostratigraphy of the non-marine Cretaceous of Argentina based on calcareous microfossils. In: INTERNATIONAL CRETACEOUS SYMPOSIUM, 3, 1989. Proceedings, Tübingen, p. 811-850.

Musacchio, E.A. 2006. Charophyta del Cretácico tardío y el Paleoceno del centro-oeste de Argentina. Revista Brasileira de Paleontología, 9(1):93-100.

Musacchio, E.A. 2010. Upper Cretaceous Lychnothamnus, Nitella and Tolypella (Charophyta) from Zampal, Argentina. Cretaceous Research, 31:en prensa.

Musacchio, E.A.; Dias Brito, D.; Maranhão, M.S. \& Suarez, J.M. 2002. Comparando carófitos (algas verdes) neocretácicos del 
Grupo Baurú (Brasil) y de Argentina. In: SIMPOSIO SOBRE EL CRETÁCICO DE AMÉRICA DEL SUR, 2, 2002. Resumos expandidos, São Pedro, UNESP, p. 329-334.

Musacchio E.A. \& Simeoni, M. 1991. Taxonomy of some Cretaceous non-marine ostracods of palaeobiogeographical interest. Neues Jahrbuch für Geologie und Palaöntologie, Abhandlungen, 180(3):349-389.

Musacchio, E.A. \& Vallati, P. 2007. Late Cretaceous non marine microfossils of the Plottier Formation at Zampal, Argentina. Cuadernos del Museo Geominero, Instituto Geológico y Minero de España, 8:273-278.

Palamarczuk, S. \& Gamerro, J.C. 1988. Grapnelispora evansii, megaspora del Cretácico Superior (?Campaniano SuperiorMaastrichtiano) de Argentina y Antártida. In: CONGRESO ARGENTINO DE PALEONTOLOGÍA Y BIOESTRATIGRAFÍA, 4, 1988. Actas, 3, Mendoza, p. 87-93.

Papú, O.H. 1989. Estudio palinológico de la Formación Paso del Sapo (Cretácico Superior), Valle Medio del Río Chubut. Granos de polen, consideraciones estadísticas, paleoecológicas y paleoambientales. Ameghiniana, 25(3):193-202.

Papú, O.H. 1990. Contribución a la palinología estratigráfica de la Formación Malargüe, Cretácico superior, sur de la provincia de Mendoza, Argentina. Parte 1: especies terrestres y de aguas continentales. Ameghiniana, 27(3-4):289-303.

Papú, O.H. 1997. Nueva especie de Grapnelispora del Campaniense-Maastrichtiense, provincia de Mendoza, Argentina. Inferencias filogenéticas, paleobiogeográficas y paleoambientales. Revista Española de Paleontología, 12(2): 197-205.

Papú, O.H. 2002. Nueva microflora de edad maastrichtiana en la localidad de Calmu-Co, sur de Mendoza, Argentina. Ameghiniana, 39(4):415-426.

Papú, O.H. \& Sepúlveda, E. 1995. Datos palinológicos de la Formación Los Alamitos en la localidad de Montoniló, Departamento 25 de Mayo, Río Negro, Argentina. Sus relaciones con unidades colindantes coetáneas. In: CONGRESO ARGENTINO DE PALEONTOLOGÍA Y BIOESTRATIGRAFÍA, 6, 1995. Actas, Trelew, v.1, p. 195-200.

Papú, O.H.; Volkheimer, W. \& Sepúlveda, E. 1988. Másulas de Salviniaceae del Cretácico tardío de Norpatagonia y sur de Mendoza. Su importancia bioestratigráfica y paleoambiental. In: CONGRESO GEOLÓGICO CHILENO, 5, 1988. Actas, Santiago, v.3, p. 167-181.

Pöthe de Baldis, E.D. 1995a. Santonian microflora of Rio Guanaco Formation (continental facies) from Rio Turbio profile, Santa Cruz Province, Argentine. In: SOUTH ATLANTIC MESOZOIC CORRELATIONS (IGCP Project $\mathrm{N}^{\circ} 381$ ), 2, 1995. Abstracts, Rio de Janeiro, p. 20.

Pöthe de Baldis, E.D. 1995b. Santonian dinoflagellates of brackishwater facies from Rio Guanaco Formation (San Martin-C profile), Santa Cruz Province, Argentina, and their relation with the Austral counterpart of the Williams Suite of temperate paleolatitudes. In: SOUTH ATLANTIC MESOZOIC CORRELATIONS (IGCP Project $\mathrm{N}^{\circ} 381$ ), 2, 1995. Abstracts, Rio de Janeiro, p. 20.

Ramos, V.A. 1981. Descripción geológica de la Hoja 33.c, Los Chihuidos Norte. Buenos Aires. Servicio Geológico Nacional, 103 p. (Boletín 182).

Regali, M.S.P. 1989. Evolução da paleoflora no Cretáceo das margens equatorial e nordeste do Brasil. Revista da Escola de Minas, 42(4):17-33.

Regali, M.S.P.; Uesugui, N. \& Santos, A.S. 1974a. Palinologia dos sedimentos meso-cenozóicos do Brasil (I). Boletim Técnico Petrobrás, 17(3):177-191.

Regali, M.S.P.; Uesugui, N. \& Santos, A.S. 1974b. Palinologia dos sedimentos meso-cenozóicos do Brasil (II). Boletim Técnico Petrobrás, 17(4):263-301.

Romero, E. 1973. Polen fósil de Nothofagus (Nothofagidites) del
Cretácico y Paleoceno de Patagonia. Revista del Museo de La Plata (Nueva Serie), Sección Paleontología, 7:291-303.

Rougier, G.W.; Chornogubsky, L.; Casadio, S.; Paez Arango, N. \& Giallombardo, A. 2009. Mammals from the Allen Formation, Late Cretaceous, Argentina. Cretaceous Research, 30:223-238.

Salgado, L.; Parras, A. \& Gasparini, Z. 2007. Un plesiosaurio de cuello corto (Plesiosauroidea, Polycotylidae) del Cretácico Superior del norte de Patagonia. Ameghiniana, 44:349-358.

Sepúlveda, E.; Papú, O.H. \& Volkheimer, W. 1989. Importancia estratigráfica y paleobiogeográfica del género Grapnelispora (Stover \& Partridge), en el Cretácico tardío del hemisferio austral. Boletim Instituto de Geociências, Publicação Especial, 7:163-171.

Stough, J.B. 1968. Palynomorphs from South America. Part 1. New Late Cretaceous palynomorphs from southern South America. The University of Kansas Paleontological Contribution Papers, 32:1-8.

Uliana, M.A. 1979. Geología de la región comprendida entre los ríos Colorado y Negro, provincias del Neuquén y Río Negro. Universidad Nacional de La Plata, Tesis Doctoral, 117 p.

Uliana, M.A. \& Dellapé, D.A. 1981. Estratigrafía y evolución paleoambiental de la sucesión Maastrichtiana-Eoterciaria del Engolfamiento Neuquino (Patagonia Septentrional). In: CONGRESO GEOLÓGICO ARGENTINO, 8, 1981. Actas, San Luis, v.3, p. 673-711.

Uliana, M.A. \& Musacchio, E.A. 1978. Microfósiles calcáreos no marinos del Cretácico Superior en El Zampal, Provincia de Mendoza, Argentina. Ameghiniana, 15(1-2):111-135.

Vallati, P. 1995. Presencia de Afropollis (Polen de Angiosperma) en el Cretácico Inferior de la Cuenca Neuquina. In: CONGRESO ARGENTINO DE PALEONTOLOGÍA Y BIOESTRATIGRAFÍA, 1995. Actas, Trelew, v.4, p. 277-290.

Vallati, P. 2001a. Middle Cretaceous microflora from the Huincul Formation ("Dinosaurian Beds") in the Neuquén Basin, Patagonia, Argentina. Palynology, 25:179-197.

Vallati, P. 2001b. Bioestratigrafía (Palinología) del Cretácico temprano y medio en Patagonia septentrional y central. Universidad Nacional de la Patagonia San Juan Bosco, Tesis Doctoral, 278 p.

Vallati, P. 2002a. Palynology of the Cerro Lisandro Formation (lower "Dinosaurian Beds") in the Neuquén Basin, west-central Argentina. Neues Jarbuch für Geologie und Paläontologie, Abhandlungen, 224(3):411-428.

Vallati, P. 2002b. Asociaciones palinológicas en el Cretácico temprano y medio de Patagonia septentrional: algunas consideraciones paleobiogeográficas. In: SIMPOSIO SOBRE EL CRETÁCICO DE AMÉRICA DEL SUR, 2, 2002. Resumos expandidos, São Pedro, UNESP, p. 157-161.

Vallati, P. 2002c. Biostratigraphy of Cretaceous non-marine sequences in Patagonia. In: EUROPEAN MEETING ON THE PALEONTOLOGY AND STRATIGRAPHY OF LATIN AMERICA, 3, 2002. Expanded Abstracts, Toulouse, p. 124-127.

Vallati, P., 2006. Las primeras angiospermas en el Cretácico de la Cuenca Neuquina (centro-oeste de Argentina): aspectos geológicos relacionados. Revista Brasileira de Paleontologia, 9(1):83-92.

Veiga, R.; Lara, M.E. \& Bruveris, P. 1999. Distribución de hidrocarburos sobre el margen externo en una cuenca de trasarco. Ejemplos en la cuenca Neuquina, Argentina. Boletín de Informaciones Petroleras, 60:142-164.

Volkheimer, W. \& Salas, A. 1975. Die älteste Angiospermen Palynoflora Argentinien von der Typus-Lokalität der Unterkretazischen Huitrín-Folge des Neuquén-beckens. Mikrofloristische Assoziation und Biostratigraphische Bedeutung. Neues Jahrbuch für Geologie und Paläontologie, Monatshefte, 7:424-436.

Received in January, 2009; accepted in May, 2010. 
Apéndice. Lista de los taxones identificados en este estudio.

Appendix. List of taxa identified in this study.

Esporas de Pteridophyta, Lycophyta, Bryophyta y de afinidad incierta

Azolla sp. (Figura 9T)

Biretisporites sp. (Figura 9E)

Camarozonosporites sp. (Figura 9l)

Ceratosporites equalis Cookson \& Dettmann, 1958 (Figura 9H)

Cicatricosisporites sp. (Figura 9M)

Clavifera triplex (Bolkhovitina) Bolkhovitina, 1966 (Figura 9F)

Cyathidites minor Couper, 1953 (Figura 9A)

Cyathidites patagonicus Archangelsky, 1972 (Figura 9B)

Cyathidites sp. (Figura 9C)

Dictyophyllidites sp. (Figura 9D)

Gabonisporis vigourouxii Boltenhagen, 1967 (Figura 7B)

Gabonisporis sp. (Figura 9P)

Gleicheniidites senonicus Ross, 1949 (Figuras 7A, 9G

Grapnelispora loncochensis Papú, 1997 (Figuras 9Q-S)

Hamulatisporis sp. cf. Rugulatisporites rionegrensis Baldoni, 1991 (Figura 9K)

Hamulatisporis sp. (Figura $9 \mathrm{~L}$ )

Klukisporites sp. (Figura 9J)

Retitriletes austroclavadites (Cookson) Döring, Krutsch, Mai \& Schulz, 1963 (Figura 9O)

Triporoletes reticulatus (Pocock) Playford, 1971 (Figura 9N)

Granos de polen de Gimnosperma

Classopollis classoides (Pflug) Pocock \& Jansonius, 1961 (Figura 7C)

Classopollis sp. (Figuras 7D-E)

Callialasporites trilobatus (Balme) Dev, 1961 (Figuras 7I, 10C)

Dacrycarpites australiensis Cookson \& Pike, 1953 (Figura 10D)

Equisetosporites cf. albertensis Singh, 1964 (Figura 7L)

Equisetosporites cf. cancellatus Paden Phillips \& Felix, 1971 (Figura 7J)

Equisetosporites multicostatus Lima, 1980 (Figura 7K)

Equisetosporites sp. (Figura $7 \mathrm{~N}$ )

Gnetaceaepollenites jansonii (Pocock) Lima, 1980 (Figuras 7P, 10E)

Microcachryidites antarcticus Cookson, 1947 (Figura 7H)

Phyllocladidites mawsonii Cookson ex Couper, 1953 (Figura 10B)

Podocarpidites cf. auriculatus Archangelsky \& Villar de Seoane, 2005 (Figura 10A)

Podocarpidites cf. marwicki Couper, 1953 (Figura 7F)

Podocarpidites rugulosus Romero, 1977 (Figura 7G)

Singhia sp. (Figura 7M)

Steevesipollenites sp. (Figura 70)

Granos de polen de Angiosperma

Afropollis zonatus Doyle, Jardiné \& Doerenkamp, 1982

Bahiasporites reticulatus Regali et al., 1974 = Confossia vulgaris Stough, 1968

cf. Beaupreaidites (Figura 10O)

cf. Propyllipollis lateflexus (Archangelsky) Baldoni \& Askin, 1993 (Figura 11D)

Clavatipollenites hughesii (Couper) Kemp, 1968

Clavamonocolpites sp. (Figura 10G)

Confossia vulgaris Stough, 1968 (Figura 8P-V)

Crassitricolporites sp. (Figura 8G-H)

Cretacaeiporites polygonalis (Jardiné \& Magloire) Herngreen, 1974

Cretacaeiporites cf. scabratus (Jardiné \& Magloire) Herngreen, 1974 (Figura 8L)

Cretacaeiporites scabratus (Jardiné \& Magloire) Herngreen, 1974 (Figuras 8M-O)

Cupanieidites? sp. (Figura 8F)

Dryadopollis sp. (Figura 8J-K)

Fraxinoipollenites fragilis Burger, 1993

Fraxinoipollenites sp. (Figura $10 \mathrm{H}$ )

Lewalanipollis cf. senectus Dettmann \& Jarzen, 1998 (Figuras 10Q-S)

Liliacidites cf. kaitangataensis Couper, 1953 (Figura 8A)

Liliacidites kaitangataensis Couper, 1953 (Figura 10F)

Liliacidites sp. (Figura 8B)

Peninsulapollis gilli (Cookson) Dettmann \& Jarzen, 1988 (Figuras 10M-N)

Peninsulapollis sp. (Figura 10P)

Propyllipollis sp. (Figura 10T)

Propyllipollis? sp. (Figura 8W)

Proteacidites cf. tenuiexinus (Stover) Stover \& Partridge, 1973 (Figura 11A)

Proteacidites sp. (Figura 11C)

Proteacidites? sp. (Figuras 11B, 8X)

Psilatricolporites sp. (Figura 8E)

Racemonocolpites? sp. (Figura $8 \mathrm{C}$ )

Retitricolporites sp. (Figura 10K)

Spinizonocolpites sp. (Figura 10I)

Striatricolporites cf. gamerroi Archangelsky, 1973 (Figura 10L)

Syncolporites? sp. (Figura 8I)

Tricesticillus tetris nov.sp. (Figuras 11E-L)

Tricesticillus americanus Stough, 1968

Tricolpites cf. reticulatus Cookson, 1947 (Figura 10J)

Tricolpites sp. (Figura 8D)

Tucanopollis crisopolensis (Regali et al.) Regali, 1989

Restos de algas

Botryococcus sp. 